\title{
versants
}

\section{La poésie ludique et les règles de la transgression}

Timothée LÉCHOT

Universités de Bâle et de Berne

\begin{abstract}
Aux XVII ${ }^{\mathrm{e}}$ et XVIII ${ }^{\mathrm{e}}$ siècles, l'énigme versifiée profite d'un vif engouement auprès des mondains, puis dans des journaux tels que le Mercure galant, le Mercure de France et le Journal helvétique. Surdéterminée par des règles, aussi bien morales qu'esthétiques, la poésie ludique constitue aussi un lieu privilégié de transgression. Nous proposons d'évaluer la manière dont l'énigme joue avec ses propres codes pour enrichir notre compréhension d'un phénomène à la fois littéraire et social.
\end{abstract}

Keywords: énigme, logogriphe, poésie, jeu, salons, journalisme.

Six pieds, ami Lecteur, composent tout mon être, Je sers à ton amusement.

Tu me cherches avidemment,

Et souvent tu me vois longtems sans me connoître.

"Énigme », Mercure de France, novembre I755, p. 74

Réponse : l'énigme.

Si le Mercure de France (I724-I79I) a le statut d'une véritable institution dans le paysage journalistique, la poésie ludique compte parmi les piliers sur lesquels repose celle-ci. Plaisants, spirituels et badins, les énigmes et logogriphes en vers incarnent, à côté d'autres pièces fugitives, le pôle divertissant du périodique français qui se définit par la variété de ses contenus. Leur publication dans chaque livraison est une nécessité à laquelle doivent se conformer les journalistes qui en sont responsables, quel que soit leur goût pour ces courtes poésies. En I756, quand une jeune Toulousaine avoue dans une lettre adressée au Mercure qu'elle déchire impitoyablement les énigmes et les logogriphes qu'elle y trouve, le rédacteur Louis de Boissy (I694-I758) répond qu'il « [pense] en secret comme elle sur le genre qu'elle condamne ", mais qu'il ne lui « est pas possible de le proscrire ${ }^{1}$ sans fâcher à la fois Paris et la Province, et sans trahir l'esprit du journal. La même né-

I Note sans signature à la suite de la lettre «A l'Auteur du Mercure ", Mercure de France, mai I756, p. 85. La jeune Toulousaine cache manifestement une plume masculine, comme on l'apprendra au mois d'août de la même année dans une "Lettre à l'Auteur du Mercure, au sujet de la Lettre \& du Logogryphe de Mlle de Car... de Toulouse, insérée dans le Mercure du mois de Mai 1756 ", pp. 76-80. 
cessité est à l'œuvre dans le Mercure suisse / Journal helvétique (I732-I782), malgré la préférence affichée des premiers éditeurs pour les matières sérieuses. Le périodique imprimé à Neuchâtel propose des énigmes et des logogriphes jusqu'en I770, de manière à satisfaire tous les goûts du public et notamment des lectrices ${ }^{2}$.

On ne saurait donc minimiser l'importance commerciale et symbolique de la poésie ludique dans de tels journaux. Elle participe de leur identité. Un lecteur le rappelle en $\mathbf{7} 763$ dans une lettre à l'auteur du Mercure de France : "Quoiqu'il en soit du mérite des énigmes, vous sçavez, Monsieur, qu'elles font une partie intégrante du Mercure depuis son institution $»^{3}$. Dans une œuvre satirique, Antoine-Joseph Gorsas (I752-I793) peut encore écrire en I786 :

Qu'on ôte du Mercure les Enigmes, les Logogryphes, les Charades, les Questions résolutives et les Bouts enrimés ;... je parie contre qui voudra, que cette planète perd les trois-quarts de son influence. - Oh ! pour moi, c'est bien décidé ; je me désabonnerois le lendemain ${ }^{4}$.

Qu'on l'approuve ou qu'on s'en fâche, la planète Mercure de France est placée sous le signe des énigmes. Cette littérature existe pourtant hors des périodiques. Sa pratique se répand et ses règles se fixent dans les cercles mondains du XVII ${ }^{\mathrm{e}}$ siècle ; la tradition orale de l'énigme se prolonge dans les salons du XVIII ${ }^{e}$ siècle, ainsi que dans les classes de rhétorique des collèges. C'est le Mercure galant (1672-I724), ancêtre du Mercure de France, qui noue en I677 un lien durable entre l'énigme et cet « immense salon de papier » qu'est le journal, publiant chaque mois de nouvelles devinettes en vers dont les réponses sont données dans la livraison suivante.

Institutionnalisée par le salon, le collège et le journal, la poésie ludique permet d'interroger les codes esthétiques et moraux qui définissent une pratique poétique et, derrière elle, les rituels d'une communauté. Plus particulièrement, nous nous demanderons ici ce que l'énigme est susceptible

\footnotetext{
2 Voir Timothée Léchot, "Le "mythe suisse" à l'épreuve des logogriphes », in Séverine Huguenin \& Timothée Léchot (dir.), Lectures du Journal helvétique I732-I782. Actes du colloque de Neuchâtel 6-8 mars 20I4, Genève, Éditions Slatkine, 20I6, pp. I75-20I.

3 «Lettre à l'Auteur du Mercure, sur les Énigmes \& les Logogryphes », Mercure de France, avril I763, t. I, p. 7I.

4 Antoine-Joseph Gorsas, L'Âne promeneur, ou Critès promené par son âne ; Chef d'œuvre pour servir d'Apologie au Goût, aux Mœurs, à l'Esprit, et aux Découvertes du siècle. Première édition revue, corrigée, et précédée d'une Préface à la Mosaïque, dans le plus nouveau goût, Pampelune : Démocrite ; Paris : chez l'Auteur, $M^{\text {de }}$. veuve Duchesne, Hardouin et Gatey, Voland, Royez ; Versailles : chez l'Auteur, I786, p. I79.

5 Christophe Schuwey, "Le Mercure galant : un recueil interactif », Cahiers du dix-septième siècle, $\mathrm{n}^{\circ} \mathrm{I} 6 / \mathrm{I}, 2015$, p. 60.
} 
de nous apprendre de la communauté formée par les lecteurs des journaux. Celle-ci demeure "virtuelle ${ }^{6}$, dans la mesure où ses membres ne se rencontrent que par le truchement du périodique, mais ses échanges n'en sont pas moins codifiés. Or la poésie ludique se caractérise souvent par la transgression des règles formulées, transgression qui redouble le plaisir du jeu. Il s'agit donc d'évaluer l'écart entre les règles et la pratique, et de vérifier si la transgression n'obéit pas elle-même à une certaine logique qui enrichirait notre compréhension d'un phénomène littéraire et social.

\section{Les règles du jeu}

Au XVIII siècle, on invoque surtout les œuvres de l'abbé Charles Cotin (I604-I68I) et du père Claude-François Ménestrier (I63I-I705) pour encadrer la pratique de l'énigme française. Dans un premier temps, nous y chercherons les règles qui définissent le fonctionnement du jeu, indépendamment de sa forme littéraire.

Cotin donne un «Discours sur les énigmes » en I646, dans son Recueil des énigmes de ce temps ${ }^{7}$. Il accorde beaucoup d'attention au choix de la chose à faire deviner. Tout ce qui relève de la nature et de l'art peut être le sujet d'une énigme, pour autant qu'il ne soit pas obscur par lui-même. Cotin rejette ainsi les devinettes qui portent sur des abstractions et, en particulier, sur des matières religieuses : la justice divine, la Vierge, un saint, etc.

Ces matieres ont-elles besoin d'être obscurcies ; et ne doit-on pas plûtost faire tout ce qui est possible afin de les éclaircir? [...] Et puis c'est en quelque manière prophaner les choses sainctes, que de les faire servir ainsi à nostre divertissement, que la pluspart ont crû le principal but de l'Enigme ${ }^{8}$.

La première barrière est donc religieuse ; un honnête homme ne la franchira pas. La seconde limitation prévient un autre excès : des réalités corporelles et basses, telles que les excréments ou les humeurs, auraient l'effet de choquer le bon goût et de « corrompre la pureté des Muses »'. Cotin refuse ainsi des énigmes sur l'ongle, par exemple, mais il tolère les parties du corps qui sont regardées sans dégoût ni impudeur : les mains, la tête, le cœur... En un mot, l'abbé mondain demande qu'on ne fasse jamais d'énigmes «sur ce

6 Voir Suzanne Dumouchel, Le Journal littéraire en France au dix-huitième siècle. Émergence d'une culture virtuelle, Oxford, Voltaire Foundation, 2016.

7 Charles Cotin, Les Énigmes de ce temps, Florence Vuilleumier-Laurens (éd.), Paris, Société des textes français modernes, 2003. Cette édition critique suit la leçon d'une réédition du recueil en I659, mais le « Discours sur les énigmes » est déjà présent dans l'édition de I646.

8 Ibid., p. 19.

9 Ibid. 
qu'on ne peut nommer honnestement ${ }^{10}$. Dans un autre ordre d'idées, Cotin se méfie des énigmes qui concerneraient plusieurs choses en même temps, comme celle de Platon sur « un Eunnuque qui frappe d'une pierre ponce une chauve-souris, laquelle estoit sur un sureau ${ }^{\text {II }}$, parce que ces choses-là n'entretiennent entre elles aucun rapport naturel.

L'énigme mobilise l'allégorie. Grâce à celle-ci, elle crée une obscurité factice autour d'une chose claire, et tout le plaisir du jeu consiste à percer cette obscurité. Là encore, Cotin prône la modération : une énigme indéchiffrable ne plairait pas. Dissociant l'énigme d'autres discours allégoriques, tels que la devise, la parabole ou l'oracle, il propose enfin une définition du genre qui restera stable au siècle suivant : "L'Enigme est un discours obscur de choses claires et connues, lequel on donne à expliquer pour l'exercice et le divertissement de l'esprit $»^{12}$. Même si Molière a laissé de Cotin l'image d'un pédant dans Les Femmes savantes, sous le nom de Trissotin, l'exercice mental que prône l'abbé n'est pas un travail laborieux réservé aux érudits, mais un délassement qui se fait en compagnie, parmi les jeux ou pendant les repas.

Comme le souligne Dominique Moncond'huy, Cotin s'érige en autorité de l'énigme, nonobstant le corpus d'écrits théoriques sur ce genre laissés par les humanistes du siècle antérieur. Revendiquant « une pratique moralement incontestable de l'énigme $»^{13}$, il dispose autour de l'énigme des bornes qui coïncident avec l'idéal social et esthétique de l'honnêteté et de la galanterie.

Cinquante ans plus tard, Ménestrier s'appuie sur la Rhétorique d'Aristote et sur Cotin pour préciser la définition de l'énigme en vers dans sa Philosophie des images énigmatiques (I694), et pour distinguer encore plus finement ces textes des autres catégories d'écritures cryptiques. En particulier, l'énigme est étrangère au mensonge :

[...] c'est un jeu d'esprit, qui cherche à donner du plaisir en donnant de la peine, parce que l'inclination, \& le desir naturel que nous avons d'aprendre, de sçavoir, \& de penetrer, fait que nous nous apliquons avec ardeur à chercher d'entrer dans les choses les plus cachées, \& quand nous y pouvons découvrir quelque jour nous sentons un veritable plaisir ${ }^{14}$.

\footnotetext{
Io Ibid.

II Ibid., p. 20. L'anecdote est présentée au livre V de La République de Platon.

I2 Ibid., p. 25.

13 Dominique Moncond'huy, "L'abbé Cotin, théoricien et praticien de l'énigme au XVII siècle ", in Stéphane Bikialo \& Jacques Dürrenmatt (dir.), L'Énigme, Rennes, Presses universitaires de Rennes, 2003, p. I6I.

I4 Claude-François Ménestrier, La Philosophie des images énigmatiques, ou il est traité des Enigmes, Hieroglyphiques, Oracles, Propheties, Sorts, Divinations, Loteries, Talismans, Songes, Centuries de Nostradamus, de la Baguette. Par le P. Cl. François Menestrier de la Compagnie de Jesus, Lyon, Hilaire Baritel, I694, p. I03.
} 
Sur le chemin qui conduit de l'obscurité de l'énigme à la clarté de la résolution, Ménestrier situe l'amusement du joueur dans le déchiffrage d'énoncés équivoques et dans le désamorçage des pièges subtils que l'auteur lui tend à chaque vers. Plus que Cotin, le père jésuite s'intéresse donc au processus herméneutique qu'implique la résolution des devinettes et qu'il associe aux joies de l'apprentissage. L'exercice de l'esprit ne sert pas seulement à susciter l'admiration, mais il a une vertu pédagogique que les amoureux de l'énigme ne manqueront jamais de rappeler au XVIII ${ }^{\mathrm{e}}$ siècle.

S'appuyant toujours sur Cotin, qu'il cite, le juriste lyonnais François Gayot de Pitaval (I673-I743) présente l'énigme écrite comme « une description qui obscurcit un sujet connu » en I72I, dans l'avertissement de son Nouveau Recueil d'énigmes ${ }^{15}$. Tout obscure qu'elle soit, la description formée par l'énigme doit être exacte et convenir uniquement à la réponse, de manière à lever toute ambiguïté après sa découverte. Comme Ménestrier, Gayot refuse d'associer l'énigme à un mensonge : c'est au contraire la vérité qui « est l'ame de l'Enigme ${ }^{16}$. La modération reste le maître-mot, puisqu'une telle poésie ne doit jamais choquer le goût ni perdre le joueur dans un dédale d'indices trop tortueux. Surtout, Gayot maintient l'interdiction de blesser une imagination délicate en lui présentant des réalités viles. Il réaffirme également l'impossibilité de consacrer des énigmes aux vérités de la religion.

En dehors de ces écrits prescriptifs, les règles de l'énigme se forgent dans les journaux, au fil du temps. Au début de l'année I733, notamment, le Mercure de France reçoit des plaintes de ses lecteurs à l'égard des énigmes et des logogriphes qui portent sur des noms de lieux ou sur des adjectifs, alors qu'on voudrait limiter les devinettes aux noms communs ${ }^{17}$. Mais globalement, les préceptes adressés aux faiseurs d'énigmes restent stables. Ils sont soit externes au genre - le cadre moral assigné à l'énigme - soit relatifs au fonctionnement interne du jeu : les lois qui garantissent au déchiffreur la possibilité d'une résolution sans mobiliser un savoir spécialisé ni éprouver une peine démesurée.

Or les infractions au code moral sont nombreuses, y compris chez les théoriciens de l'énigme que nous avons évoqués. Soit dans l'édition princeps de I638, soit dans la réédition de I646, Cotin met la décence et le bon goût entre parenthèses pour consacrer des énigmes au pet, aux fesses et aux tétons. Gayot s'accordera les mêmes licences dans sa compilation, reprodui-

I5 François Gayot de Pitaval, Nouveau Recueil d'énigmes. Dédié à Son Altesse Serenissime Monseigneur le Prince de Conty. Nouvelle Edition corrigée \& augmentée de deux cens Enigmes, Paris, Théodore Le Gras, I72I, rom. La première édition du recueil, en I7I7, contient un avertissement moins étendu.

I6 Ibid.

I7 «Avertissement », Mercure de France, janvier I733, rom. 
sant « deux Enigmes sur une ordure $»^{18}$ qui étaient trop ingénieuses pour être ignorées. Hors des recueils, on se permet encore plus d'entorses. Le poète libertin Jean-Baptiste de Grécourt (I684-I743) laisse dans ses Euvres diverses une énigme sur le "pot à chier », expression qu'il cache derrière le nom du médecin "Hipocrate», son anagramme ${ }^{19}$. On note aussi qu'une pièce anonyme du Journal helvétique fait deviner l'« étron » à l'aide du mot " trône ", par le déplacement de la lettre $e$ :

Il n'est pas de mortel qui ne me fasse naitre.

Et je puis me vanter de mon utilité.

Aussi-tôt cependant que je viens à paroître

Avec soin je suis écarté.

$[\ldots]$

Telles sont des humains les contrarietés!

Mais en voici de plus grandes encore.

Qu'on me mette la tête aux pieds :

L'on me respecte, l'on m'honore.

Et j'excite les vœux des plus grands des humains.

Autrefois méprisé par les anciens Romains [,]

J'ai su leur donner des entraves

Et de ces fiers vainqueurs faire de vils esclaves ${ }^{20}$.

Dans ces textes, la transformation burlesque de réalités nobles en choses abjectes a une vocation ludique. L'énigme ouvre un espace littéraire à ce qui relève de l'indicible dans le champ des belles-lettres et dans un univers social dont les interactions sont régies par la politesse des mœurs et du langage. Loin d'être épargnée comme l'auraient souhaité Cotin et Gayot, la religion est la plus flagrante victime des énigmes à caractère provocateur. Hors de la littérature clandestine, on ne connaît pas d'autre genre qui permette, par exemple, de décrire l'union d'Adam et d'Ève sous le signe de l'inceste, de l'homosexualité et de l'hermaphrodisme ${ }^{21}$.

I8 François Gayot de Pitaval, éd. cit., rom.

19 Jean-Baptiste de Grécourt, "Anagramme », Euvres diverses de Grécourt ; nouvelle édition, soigneusement corrigée, E augmentée d'un grand nombre de Piéces qui n'avoient jamais été imprimées, Luxembourg, I76I, t. 3, p. I2I.

20 "Logogriphe », Nouveau Journal helvétique, Neuchâtel, février I770, pp. 255-256.

2I Voir Timothée Léchot, "Je porte dans mon sein mon ennemi mortel". L'énigme et le sacré au XVIII ${ }^{e}$ siècle ", à paraître dans les actes du $6^{\mathrm{e}}$ colloque international du Laboratoire d'études et de recherches interdisciplinaires et comparées (LERIC) «L'imaginaire ludique : création et enjeux » (Sfax, décembre 2016). 


\section{Les règles du genre}

Le jeu de la transgression se prolonge à l'égard d'une autre catégorie de règles, celles qui définissent l'énigme en tant que genre poétique et œuvre esthétique. Certes, l'énigme n'est jamais l'objet d'une réflexion développée chez les grands poéticiens des XVII ${ }^{\mathrm{e}}$ et XVIII ${ }^{\mathrm{e}}$ siècles (Boileau, Fénelon, Dubos ou Batteux) et personne ne doute qu'elle forme un genre mineur au sein de la poésie française. À ce titre, elle hérite cependant des règles de la versification classique qui fixent les caractéristiques rythmiques, métriques et sonores du vers, et qui exigent le recours à un lexique châtié. Mais ce qui frappe, depuis Cotin, c'est que la définition littéraire de l'énigme repose sur des figures de rhétorique. Pour l'auteur du Recueil des énigmes de ce temps, c'est avant tout une allégorie tissée de métaphores, au sens où toutes les métaphores que déploie l'énigme forment un système de représentation cohérent, une fois la réponse découverte. Cotin note aussi l'importance de l'antithèse dans l'énigme, parce que celle-ci s'appuie généralement sur la confrontation de notions contradictoires, comme on peut l'illustrer à travers cette minuscule pièce du Mercure de France:

Quel singulier destin!

Hier j'étois demain ${ }^{22}$.

Aucun doute pour Cotin : mobilisant les ressources de la rhétorique, de la langue et du vers, "il faut que ce genre de Poésie plaise »" qu'il plaise avant qu'on ait déduit la solution et qu'il plaise encore après qu'on l'a trouvée.

Ménestrier, quant à lui, regarde la prosopopée comme une figure typique de l'énigme : les devinettes « les plus vives \& les plus agréables ${ }^{24}$ sont celles où la réponse prend la parole à la première personne pour donner des indices aux lecteurs sur ses différentes propriétés. La métaphore reste centrale : tout l'art du faiseur d'énigme consiste à déguiser une chose avec d'autres choses qui lui ressemblent (les indices), mais qui ne se ressemblent pas entre elles et qui créent ainsi une description équivoque. L'alliage de la similitude et de la dissemblance provoque un effet qui tient du " merveilleux $»^{25}$ selon La Philosophie des images énigmatiques, dans la mesure où ce qu'il est impossible de concevoir au niveau de la description se résout comme par enchantement dans la réponse.

22 "Autre [énigme] », Mercure de France, juillet I768, t. 2, p. 68. Réponse : aujourd'hui.

23 Chares Cotin, op. cit., p. 27.

24 Claude-François Ménestrier, op. cit., p. IO9.

25 Ibid., p. 120. 
Tributaire à la fois de Cotin et de Ménestrier, Gayot redonne la liste des figures propres aux « Enigmatistes $~_{26}$, érigeant pour sa part l'antithèse en ressort favori du genre. Il entérine en outre une loi que ses prédécesseurs suggéraient déjà, à savoir la nécessité de recourir au vers plutôt qu'à la prose : «Une tragédie en prose seroit un monstre ; une Enigme en prose ne sçauroit plaire aux connoisseurs. La poësie est affectée à certains ouvrages d'esprit $»^{27}$.

Possédant des règles relatives au contenu et à la forme, l'énigme se présente bien, dès le XVII ${ }^{\mathrm{e}}$ siècle, comme un genre littéraire à part entière, situé à l'intersection d'au moins deux catégories de textes : les genres mondains que pratiquent les salonniers et les écritures emblématiques ou codées dont traite Ménestrier ${ }^{28}$. Au XVIII ${ }^{\mathrm{e}}$ siècle, l'énigme rejoint un troisième ensemble, celui des "pièces fugitives " qui forment une rubrique distincte dans des journaux comme le Mercure de France, et où les jeux littéraires côtoient aussi bien des courtes poésies que des mémoires savants, des comptes rendus de séances académiques, des courriers de lecteurs, etc. ${ }^{29}$

C'est là que se développe le logogriphe, sous-genre particulier d'énigmes versifiées qui élève d'un degré la complexité du jeu. Il apparaît dans le Mercure de France dès le mois de décembre $1727^{30}$. Tandis que l'énigme désigne traditionnellement une chose, le logogriphe concerne un signifiant : le mot lui-même. Dans un logogriphe canonique, le joueur est confronté à plusieurs courtes énigmes qui portent sur des transpositions anagrammatiques du mot à deviner, anagrammes qui lui permettent ensuite de recomposer la réponse. C'est ainsi qu'on passe de trône à étron dans l'exemple cité plus haut ou, dans un autre texte du Mercure, du mot athéisme au mot catéchisme, le premier étant l'anagramme partielle du second ${ }^{31}$.

Par son recours à l'anagramme, le logogriphe enrichit encore le bagage rhétorique de l'énigme, mais il parasite le processus d'allégorisation jugé essentiel par les théoriciens. Il brise en effet le lien naturel entre les indices et la réponse, ce qui multiplie les possibilités de rapprochements incongrus entre des réalités qui ne partagent rien en dehors des lettres ou des syllabes dont on se sert pour les exprimer. En outre, en tant qu'énigme composée de petites énigmes, le logogriphe compromet l'unité du texte et il menace l'équilibre fragile, demandé par Cotin et ses successeurs, entre la peine du

26 François Gayot de Pitaval, op. cit., rom.

27 Ibid.

28 Voir Georges Couton, Écritures codées. Essai sur l'allégorie au XVII siècle, Paris, Aux amateurs de livres, 1990 ; et Anne-Elisabeth Spica, Symbolique humaniste et emblématique. L'évolution des genres (I580-I700), Paris, Honoré Champion, 1996.

29 Voir Nicole Masson, La poésie fugitive au XVIII siècle, Paris, Honoré Champion, 2002.

30 "Logogryphe ", Mercure de France, décembre i727, t. I, pp. 2988-2989.

3I Voir Timothée Léchot, «"Je porte dans mon sein mon ennemi mortel”. L'énigme et le sacré au XVIII siècle», art. cit. 
décryptage et le plaisir de la résolution. Les logogriphes « embarrassent \& fatiguent trop l'esprit ", regrette un compilateur du milieu du XVIII siècle ; ils ont souvent le défaut d'« embrasser trop de choses", de "renfermer trop d'idées ", d'être "presque inexplicables "; beaucoup d'entre eux ne sont «en aucune façon agréables $»^{32}$. Fils naturel de l'énigme, mais illégitime, le logogriphe naît d'une entorse aux principes du genre, motif supplémentaire de suggérer que la subversion ou la redéfinition des règles participe intimement du plaisir ludique.

Or ce que l'énigme et le logogriphe subvertissent par-dessus tout, ce sont les frontières génériques. L'énigme n'est pas seulement un genre, mais un mode du discours symbolique qui peut être insufflé dans d'autres genres, voire dans des formes fixes: Cotin pratique abondamment l'art du « sonnet énigmatique » dans son recueil. De même, la proximité de l'énigme avec l'épigramme saute aux yeux de tous les commentateurs, parce que les deux genres se prêtent efficacement à l'usage de la pointe. Cotin se moque par exemple du gazetier de Bruxelles dans une énigme qui est tout à la fois un sonnet et une épigramme ${ }^{33}$. De l'épigramme, l'énigme dérive vers le pamphlet. En I79o, le royaliste Jean-Gabriel Peltier (I760-I825) publie une longue énigme sanglante qui, pour faire deviner le nom de Mirabeau, décrit la naissance d'un monstre dans la boue des Enfers, sous l'œil infâme de ses parents, l'Envie et Mégère ${ }^{34}$.

Au-delà des croisements génériques motivés par la pointe, énigmes et logogriphes prennent toutes sortes d'habits différents dans les périodiques du XVIII ${ }^{e}$ siècle. Un lecteur du Journal helvétique se propose d'introduire la pratique de l'« Ode énigmatique ", publiant à titre de modèle une énigme qui, comme l'ode, est structurée en strophes régulières ${ }^{35}$. De son côté, le Mercure de France donne à l'occasion des «Stances logogriphiques ${ }^{36}$. Les journaux proposent enfin de nombreuses « énigmes en vaudeville » ou « logogriphes en chanson", avec l'indication de l'air sur lequel ils doivent être entonnés ${ }^{37}$.

Surtout, on recense une infinité d'hybridations au sein même de la poésie ludique : « énigmes logogriphiques », « logogriphes énigmatiques ", « énigmes en logogriphe »... Ces textes troublent, non plus la distinction de catégories textuelles hétérogènes, mais celle du genre et de l'espèce : si le lo-

32 Pierre-Charles Berthelin, «Avertissement », Recueil d'énigmes et de quelques logogriphes. Par M. l'Abbé Berthelin, Paris, Antoine Boudet, 1746, p. VI.

33 Charles Cotin, op. cit., énigme LIX.

34 "La Race de Mégère. Charade ", Les Actes des Apôtres, commencés le jour des Morts, E finis le jour de la Purification, Paris, « l'an de la liberté o » [1790], t. I, pp. I47-I49.

35 "Lettre écrite aux Editeurs », Mercure suisse, mars I733, pp. 9I-IOI.

36 Mercure de France, avril I75I, pp. I07-I08. Le texte est signé « Bruno du Puget » et daté de "Cuers en Provence, le 7 Février I75I ".

37 Voir, par exemple, les livraisons du Journal helvétique de novembre 1753, août 1754, septembre 1754 et février 1755 . 
gogriphe est une déclinaison de l'énigme, il est par définition énigmatique. Or la confusion atteint son paroxysme lorsque le Mercure de France publie un (ou une) « énigmogogryphe » en I757 :

LECTEUR charmant, brave, mais trop volage,

Je ne suis point de ton pays,

Et ne veux sur mon tout tenir autre langage.

Vole toujours de Laïs en Laïs,

Tandis que pour jamais en amour je m'engage,

Et que, sans rien changer, uniment je le dis ;

Je te le dis, Nymphe charmante:

Mon nom est familier, n'en prends aucun effroi ;

L'honnêteté réside en moi,

Et suit ton nom, à bon droit je m'en vante :

Du reste, je ne dirai rien ;

Je suis presqu'aussi long que le plus long rosaire,

Et de ma dixaine derniere

Le Public se passera bien ${ }^{38}$.

À mi-chemin de l'énigme et du logogriphe, cette poésie est considérée comme une "piece Hermaphrodite " par le rédacteur du Mercure ${ }^{39}$. Jeu sur le genre (littéraire et grammatical), elle cache une réponse pour le moins originale et difficile à découvrir : le mot latin Constantinopolitanensibus. Ce qui la rapproche du logogriphe, c'est l'usage d'anagrammes : l'adjectif constant, la nymphe Ino et l'adjectif poli. En revanche, les dix dernières lettres du mot ne sont l'objet d'aucun indice, empêchant d'y voir un logogriphe en bonne et due forme.

De même, lorsqu'on l'envisage du point de vue de la versification, l'énigme est loin de se cantonner dans une pratique étroitement normée. Des mètres rares sont expérimentés, comme le trissyllabe dans une énigme de 1746 sur le « nez » qui commence ainsi :

Court ou long,

C'est selon

Que mon Maître

Me fait naître,

Ecrasé,

Malposé,

Quelle forme

Plus difforme ${ }^{40}$ !

38 «Enigmogogryphe », Mercure de France, juin I757, t. 2, p. 66.

39 [Note précédant la pièce], ibid., p. 65.

40 «Énigme», Mercure de France, juin I746, t. I, p. I43. 
Comme le nez, la poésie peut être « écrasée », voire « difforme » dans une énigme. Celle-ci va parfois sacrifier la grâce du vers français traditionnel à l'efficacité d'une liste où les indices se succèdent sans structure syntaxique. Cette approche inhabituelle de la versification caractérise notamment un logogriphe de 1756 sur le mot privilège où les anagrammes sont simplement juxtaposées à l'intérieur des vers. On y lit dix-huit pentasyllabes comme les suivants :

Livrée, ire, livre,

Père, leger, ivre,

Pilier, rive, pré,

Grive, plier, té, $[\ldots]^{41}$.

À la même époque, on teste aussi les pièces monorimes, comme dans cette autre énigme du Mercure de France qui désigne le peigne et dont voici les premiers vers :

Je tiens de la frivolité,

Je plais par ma légéreté;

De m'avoir, on est entêté :

On vante ma commodité,

J'ai pourtant peu d'utilitéét

Bien sûr, l'écart avec les normes littéraires et esthétiques ne réjouit pas tous les lecteurs des journaux. C'est la principale cause du discrédit dans lequel tombent l'énigme et, en particulier, le logogriphe auprès des plus sceptiques. Quoique amateur d'énigmes, La Condamine lui-même prend la parole dans le Mercure de France ${ }^{43}$ pour dénoncer, entre autres vices littéraires, une pratique courante du logogriphe qui consiste à numéroter les lettres de la réponse et des anagrammes qui la composent, si bien que des hémistiches entiers sont uniquement composés de chiffres arabes à l'exemple de cet alexandrin : « $8 \& 9,3,4$, I, je suis un bruit flateur $»^{44}$. Un éditeur du Mercure stigmatise quant à lui l'irrespect des auteurs d'énigmes pour les conventions les plus élémentaires de la versification. Pour lui, en I756, la décadence de l'énigme est symptomatique du déclin de la poésie. Il

4I "Logogryphe», Mercure de France, avril I756, t. I, p. 93.

42 "Enigme », Mercure de France, octobre I758, t. I, p. 63.

43 Cité par Jean-François Marmontel à l'article Énigme \& Logogriphe des Éléments de littérature [1787], Sophie Le Ménahèze (éd.), Paris, Éditions Desjonquères, 2005.

44 Louis Chapat, "Logogriphe ", Petit Recüeil de poësies par Louis Chapat conseiller de cour au service de Sa Majesté le Roy de Prusse, Berlin, Jean Grynæus, I743, p. I09. La réponse du logogriphe est « Minotaure » et la présente anagramme désigne le « renom ». 
cite quelques mauvais vers d'un logogriphe reçu, qu'il se refuse à publier, puis il les commente ainsi :

Nous les citons exprès pour faire voir à quel point on porte aujourd'hui l'oubli des regles, \& pour justifier les plaintes que nous avons faites à ce sujet dans le second volume de Janvier. Nous sçavons qu'une Enigme ou qu'un Logogryphe n'exigent pas une exactitude scrupuleuse, mais on doit au moins observer la mesure. Souvent on n'est pas plus régulier pour des piéces d'un genre plus élevé. On y rime au hasard une prose des plus familieres, \& l'on lui donne fiérement le nom de Stances, quelquefois même le titre pompeux d'Ode ${ }^{45}$.

Pour des hommes de lettres comme l'auteur de cette note, l'énigme incarne un genre, sinon contaminateur, du moins révélateur de l'état alarmant de la poésie française. Considérée sous cet angle, la poésie ludique n'a plus rien d'anodin. Discours emblématique, elle devient elle-même, pour ainsi dire, un emblème de la licence.

\section{L'esprit critique et l'abus de confiance}

L'énigme est à la fois un genre surdéterminé par des règles et un lieu privilégié de transgression. Ce paradoxe peut l'ériger en menace pour le goût, voire pour les mœurs, ce dont les auteurs se montrent parfois conscients. En I73I, quatre ans après l'émergence du logogriphe dans le Mercure de France, un «quatrain énigmatique » a pour réponse « le Logogryphe du Mercure » :

Ung Monstre élapsera de la Rive de Nil,

Vers l'an trente après mil sept cent qu'on nombrera ;

Neuf pieds, une aîle et grife, et double os il aura,

Ung Dieu l'ostendera sur l'horison de $\mathrm{Cil}^{46}$.

Pastichant le moyen français de la Renaissance, le texte est signé : «Par un Eleve de Nostradamus ». Il joue sur la proximité entre l'énigme et la prophétie. Dans cette devinette autoréférentielle, le logogriphe est métaphorisé sous les traits d'un oiseau de mauvais augure, un " monstre " griffu et terrifiant. S'il s'échappe du Nil, c'est qu'il doit sa naissance à cet autre monstre qu'est le Sphinx du mythe d'Edipe. Ses « neuf pieds » sont les neuf lettres de « logogrife », qui contiennent effectivement une " grif[f]e », une aile (la lettre $l$ ) et deux os (la lettre $o$ ). Le dieu malfaisant qui va libérer cette créa-

45 [Note sans titre], Mercure de France, mars I756, pp. 89-90.

46 "Quatrain énigmatique », Mercure de France, août I73I, pp. 194I-I942. 
ture vers I730 est bien sûr Mercure, c'est-à-dire le journal. Ici, tout concourt à la peinture de la difformité. La décomposition anagrammatique du mot crée une chimère, tandis que la langue gothique mime la complaisance du logogriphe pour l'obscurité. Loin de condamner ce genre, le disciple anonyme de Nostradamus rend hommage aux vertus divertissantes d'une poésie décomplexée.

De ces exemples, on conclurait trop vite que tout est permis dans le registre ludique. Première hypothèse, il semble que les différents niveaux de prescription - le cadre moral, la distinction des genres, le respect de la versification - ne soient jamais tous brouillés dans un même texte. Le logogriphe de l'étron et l'« énigmogogryphe » peuvent respectivement paraître indélicat et obscur, mais leur versification hétérométrique se veut irréprochable. À l'inverse, les différents exercices de style cités (énigme monorime, trissyllabe, logogriphe en forme de liste) n'ont rien de scabreux sur le plan du contenu et, expériences formelles à part, ce sont des devinettes parfaitement orthodoxes sur le nez, le privilège ou le renom.

Seconde hypothèse, la plupart des transgressions restent acceptables par le public, et même bienvenues, mais seulement dans la mesure où elles ne génèrent pas de frustration. Si le plaisir de la mystification est intrinsèque à l'énigme, il faut néanmoins que le « joueur joué » puisse se dédommager par un quelconque plaisir.

Cotin publie dans certaines éditions de son recueil une « énigme de raillerie » qui ne désigne rien, mais dont l'imposture est immédiatement signalée, sans laisser le temps au lecteur d'être pris au piège :

Ie caresse le peuple, \& suis chery des Princes, Ie suis esclave \& Roy de cent mille Prouinces, Tantost ie regne en femme, \& tantost en Amant, Qui pretend m'expliquer perd sa Philosophie, Veux-tu sçavoir, Montreuil, ce que ie signifie? Ma foy, rien, ie ne suis qu'un sizain seulement ${ }^{47}$.

Dominique Moncond'huy remarque que ce texte « dénonce à sa manière un certain visage de la pratique du genre $»^{48}$. Cotin est en effet sensible aux tensions et aux risques de cette activité. La présence d'une telle " raillerie » invite, dans un cadre mondain, à une forme d'autodérision qui protège le joueur d'un engouement déraisonné pour un tel divertissement. Le destinataire Montreuil, avide de connaître le mot de l'énigme, saura désormais prendre un recul critique sur les défis qu'on présentera à son esprit.

47 Charles Cotin, «Enigme 73", Recveil des énigmes de ce temps, Paris, Tovssaint Qvinet, I646, t. 2, 82. L'expression « énigme de raillerie » apparaît dans la table des réponses, en fin de volume. 48 Dominique Moncond'huy, art. cit., p. I67. 
Un manquement aux lois de l'énigme peut donc avoir l'effet paradoxal de réaffirmer un code social. Longtemps après Cotin, dans les années I770, le poète Jean-Baptiste Gresset (I709-1777) aurait donné une même leçon de sociabilité à un groupe de lettrés, dénonçant cette fois l'ennui que l'énigme peut provoquer lorsqu'on en abuse. Sans doute apocryphe, mais significative, l'anecdote est rapportée par Jean-François de La Harpe :

Il [Gresset] était dans une société où l'on proposait souvent des énigmes, l'un des grands travaux du bel-esprit de province. Gresset qui en était las, apporta un jour la sienne qui n'avait que deux vers :

Je suis un ornement qu'on porte sur la tête, Je m’appelle chapeau : devine, grosse bête.

Tout le monde se mit à rire ; mais quelqu'un qui ne riait pas, après avoir rêvé quelque temps très-sérieusement, se leva en criant, je l'ai trouvé, je l'ai trouvé; c'est une perruque ${ }^{49}$.

Subvertissant l'énigme, Gresset rappelle qu'elle doit constituer un jeu plaisant et un trait d'esprit suscitant l'admiration. Dans cette anecdote, l'ingénu qui s'obstine à résoudre le canular n'est pas seulement un provincial mal dégrossi. C'est aussi un homme qui a oublié les fondements essentiellement ludiques de l'énigme.

Or la communauté que forment les lecteurs d'un journal n'est évidemment pas homologue à celle d'une société littéraire. Ses membres anonymes interagissent par écrit. Le rythme de ces échanges est dicté par la périodicité de la publication, généralement limitée à une ou deux livraisons par mois pour les journaux qui proposent des énigmes. En outre, les abonnés ont un engagement financier dans cette communauté, le prix d'un périodique n'étant pas négligeable pour une bourse moyenne, même quand des groupes de lecteurs s'associent pour acheter un exemplaire commun. Ces données ont un impact sur les attentes à l'égard des jeux littéraires. D'un côté, le public dispose d'un laps de temps confortable pour décrypter les énigmes, en attendant la solution qui est presque systématiquement donnée dans la livraison suivante. Dès lors, des devinettes trop faciles sont susceptibles de décevoir, ce qui explique peut-être le succès des textes plus complexes comme certains logogriphes. D'un autre côté, les amateurs d'énigmes ne manquent jamais de signifier leur colère devant une devinette incompréhensible.

49 Jean-François de La Harpe, Correspondance littéraire, adressée à son Altesse Impériale M. ${ }^{g r}$ le Grand-Duc, aujourd'hui Empereur de Russie, et à M. le Comte André Schowalow, Chambellan de l'Impératrice Catherine II, depuis I774 jusqu'à I789 ; Par Jean-François Laharpe, Paris, Migneret, t. 2, I8OI, p. I26. 
Dans le Mercure de France de mai I730, un homme adresse une lettre au journal pour raconter le trouble jeté dans son ménage par le trente-etunième vers d'un logogriphe, sujet d'une dispute entre sa femme et lui. Portant sur un mot latinisé, ce vers a provoqué la colère de l'épouse, parce que, ne maîtrisant pas le latin, elle s'est sentie exclue de la communauté des déchiffreurs d'énigmes, quand bien même les femmes sont « les juges naturels de ces sortes d'Ouvrages $»^{50}$. Cette affaire, qui est peut-être une simple plaisanterie, encourage le Mercure à maintenir son cap entre la frivolité et le pédantisme, les énigmes et les logogriphes ayant pour fonction de satisfaire et sans doute de fidéliser les lecteurs non savants.

Ce qu'on ne pardonne pas, ce sont les énigmes qui, sans se présenter comme des railleries, ne recèlent aucune réponse. Les efforts du joueur sont alors vains et le pacte de confiance qui le lie à son journal est douloureusement rompu. Au xix siècle, on rapporte une autre anecdote qui, toute factice qu'elle paraisse, a l'intérêt d'associer l'amour de l'énigme aux passions les plus violentes :

Le $\mathrm{I}^{\mathrm{er}}$ avril $\mathrm{I} 76 \mathrm{o}$, tout Paris fut en émoi. On se cherchait, on s'interrogeait avec inquiétude, on se quittait avec douleur ; c'était un deuil général, une calamité publique : Qu'était-ce donc ? C'est que la charade du Mercure était introuvable, c'est que les plus habiles s'y brisaient le front : [...] c'était un piège, un guet-apens tendu par le Mercure à la bonne foi de ses abonnés. L'indignation fut à son comble : on assure qu'un marquis se déclara le chevalier du public outragé, et se fit tuer en duel par un rédacteur du Mercure $^{55}$.

Se provoque-t-on en duel pour des jeux littéraires ? Certainement pas, mais un autre témoignage, plus crédible, atteste l'effervescence du public allemand, en 1784 , autour de deux devinettes insolubles. On lit dans le Journal politique de Bruxelles :

Des Enigmes ont occupé cette année une partie du Public en Allemagne. [...] Les explications [proposées] étoient plus ridicules les unes que les autres. Des gens de la plus haute condition s'en sont mélés. Il est vraisemblable que ces Enigmes n'avoient point de solution, \& qu'un plaisant a voulu jouer le Public, pour prouver qu'il n'est pas encore temps de crier contre le trop de lumieres ${ }^{52}$.

50 "Lettre écrite de Chambery, à l'Auteur du Mercure de France, le 26. Mars I730 ", Mercure de France, mai I730, p. 942.

5I "Amusement de l'esprit », Dictionnaire de la conversation et de la lecture, Paris, Belin-Mandar, t. 2, I832, p. II4.

52 «Allemagne », Journal politique de Bruxelles, $\mathrm{n}^{\circ}$ 39, 25 septembre $\mathrm{I784}$, pp. I48-I50. 
Selon le journaliste, une énigme qui nierait sa principale règle de jeu ne commettrait pas cette infraction gratuitement. L'énigme sans réponse devient une énigme au second degré : le travail d'interprétation se déplace du texte au geste critique qui motive la transgression.

Ainsi, dans le domaine de la poésie ludique, la transgression s'inscrit dans la logique même du jeu, qui est de surprendre, d'instruire ou d'amuser. Fondée sur l'idée d'obscurcir artificiellement ce qui est naturellement clair, l'énigme se prête bien à interroger les conventions les plus admises, à commencer par les normes littéraires et sociales.

Si le plaisir de jouer implique en même temps « un désir de liberté et une passion pour les règles ", alors « la poésie est naturellement un jeu » et l'expression de "poésie ludique » devient pléonastique ${ }^{53}$. Il serait tentant, dans cette perspective, de regarder les faiseurs d'énigmes comme les oulipiens des XVII ${ }^{\mathrm{e}}$ et XVIII ${ }^{\mathrm{e}}$ siècles. On trouve d'ailleurs des tautogrammes et des lipogrammes - parmi les contraintes chères aux oulipiens - chez un littérateur de 1725 qui pratique l'énigme, l'abbé Louis de Court (né vers I658, mort en I732) ${ }^{54}$. Cependant, l'exploration des contraintes n'a pas encore, sous l'Ancien Régime, une dimension formaliste et réflexive généralisée. Si les lecteurs et les contributeurs du Mercure de France y découvrent un potentiel heuristique, rafraîchissant et parfois insolent, les théoriciens s'attachent plutôt à donner au genre la dignité qu'on lui conteste, en insistant sur ses origines antiques et en affermissant ses assises rhétoriques.

\section{Bibliographie}

Berthelin, Pierre-Charles, Recueil d'énigmes et de quelques logogriphes. Par M. l'Abbé Berthelin, Paris, Antoine Boudet, I746.

Chapat, Louis, Petit Recüeil de poësies par Louis Chapat conseiller de cour au service de Sa Majesté le Roy de Prusse, Berlin, Jean Grynæus, 1743.

Cotin, Charles, Les Énigmes de ce temps [1659], Florence Vuilleumier-Laurens

(éd.), Paris, Société des textes français modernes, 2003.

—. Recueil des énigmes de ce temps, Paris, Tovssaint Qvinet, 1646.

53 Jean-Pierre Étienvre cité et traduit de l'espagnol par Samuel Fasquel, Quevedo et la poétique du burlesque au XVII esiècle, Madrid, Casa de Velázquez, $201 \mathrm{I}$, p. 155.

54 Notamment, bien avant La Disparition (1969) de Georges Perec, une « Lettre seconde dans laquelle on ne fait point entrer la voyelle E " (Louis de Court, Varietez ingenieuses ou recueil et melange de pieces serieuses et amusantes. Par M. D. **** Academicien, Paris, Christophe David, I725, pp. 263-266). Perec se souviendra de ce texte. Voir Alain Chevrier, «L'abbé Louis de Court, Variétés ingénieuses (1724). Une lettre lipogrammatique en $e$ mentionnée par Georges Perec ", in David Bellos, Marcel Bénabou, Bernard Magné (dir.), Georges Perec et le renouveau des contraintes, Formules. Revue des littératures à contraintes, $\mathrm{n}^{\circ}$ 6, 2002, pp. I08-I09. 
Court (de), Louis, Varietez ingenieuses ou recueil et melange de pieces serieuses et amusantes. Par M. D. *** Academicien, Paris, Christophe David, I725.

Couton, Georges, Écritures codées. Essai sur l'allégorie au XVII siècle, Paris, Aux amateurs de livres, 1990.

Dumouchel, Suzanne, Le Journal littéraire en France au dix-huitième siècle. Émergence d'une culture virtuelle, Oxford, Voltaire Foundation, 2016.

Gayot de Pitaval, François, Nouveau Recueil d'énigmes. Dédié à Son Altesse Serenissime Monseigneur le Prince de Conty. Nouvelle Edition corrigée \& augmentée de deux cens Enigmes, Paris, Théodore Le Gras, I72I.

Gorsas, Antoine-Joseph, L'Âne promeneur, ou Critès promené par son âne ; Chef d'œuvre pour servir d'Apologie au Goût, aux Mœurs, à l'Esprit, et aux Découvertes du siècle. Première édition revue, corrigée, et précédée d’une Préface à la Mosaïque, dans le plus nouveau goût, Pampelune, Démocrite ; Paris, chez l'Auteur, $M^{\text {de }}$. veuve Duchesne, Hardouin et Gatey, Voland, Royez ; Versailles, chez l'Auteur, I786.

Grécourt (de), Jean-Baptiste, Euvres diverses de Grécourt ; nouvelle édition, soigneusement corrigée, E augmentée d'un grand nombre de Piéces qui n'avoient jamais été imprimées, Luxembourg, I76I.

La Harpe (de), Jean-François, Correspondance littéraire, adressée à son Altesse Impériale M..$^{g r}$ le Grand-Duc, aujourd'hui Empereur de Russie, et à M. le Comte André Schowalow, Chambellan de l'Impératrice Catherine II, depuis I774 jusqu'à I789; Par Jean-François Laharpe, Paris, Migneret, I80I-I807.

Léchot, Timothée, «Le "mythe suisse” à l'épreuve des logogriphes », in Séverine Huguenin \& Timothée Léchot (dir.), Lectures du Journal helvétique I732-I782. Actes du colloque de Neuchâtel 6-8 mars 20I4, Genève, Éditions Slatkine, 20I6, pp. I75-20I.

-. " "Je porte dans mon sein mon ennemi mortel". L'énigme et le sacré au XVIII ${ }^{\mathrm{e}}$ siècle ", L’imaginaire ludique: création et enjeux (Sfax, décembre 20I6), Actes $d u \sigma^{e}$ colloque international du Laboratoire d'études et de recherches interdisciplinaires et comparées (LERIC) [à paraître].

Marmontel, Jean-François, Éléments de littérature [I787], éd. Sophie Le Ménahèze, Paris, Éditions Desjonquères, 2005.

Masson, Nicole, La Poésie fugitive au XVIII siècle, Paris, Honoré Champion, 2002.

Ménestrier, Claude-François, La Philosophie des images énigmatiques, ou il est traité des Enigmes, Hieroglyphiques, Oracles, Propheties, Sorts, Divinations, Loteries, Talismans, Songes, Centuries de Nostradamus, de la Baguette. Par le P. Cl. François Menestrier de la Compagnie de Jesus, Lyon, Hilaire Baritel, I694.

Mercure de France, Paris, I724-I79I.

Mercure suisse / Journal helvétique, Neuchâtel, I732-I782. 
Moncond'huy, Dominique, "L'abbé Cotin, théoricien et praticien de l'énigme au XVII siècle ", in Stéphane Bikialo \& Jacques Dürrenmatt (dir.), L'Énigme, Rennes, Presses universitaires de Rennes, 2003, pp. 157I7I.

Schuwey, Christophe, «Le Mercure galant : un recueil interactif », Cahiers $d u$ dix-septième siècle, $\mathrm{n}^{\circ} \mathrm{I} 6 / \mathrm{I}, 2015, \mathrm{pp}$. 48-62.

Spica, Anne-Elisabeth, Symbolique humaniste et emblématique. Lévolution des genres (I580-I700), Paris, Honoré Champion, 1996. 